\title{
Kernos
}

Revue internationale et pluridisciplinaire de religion grecque antique

3| 1990

Varia

\section{Marie Delcourt, dix ans déjà}

Hommage à Marie Delcourt

André Motte

\section{(2) OpenEdition \\ 1 Journals}

\section{Édition électronique}

URL : http://journals.openedition.org/kernos/967

DOI : $10.4000 /$ kernos.967

ISSN : 2034-7871

Éditeur

Centre international d'étude de la religion grecque antique

Édition imprimée

Date de publication : 1 janvier 1990

ISSN : 0776-3824

Référence électronique

André Motte, « Marie Delcourt, dix ans déjà », Kernos [En ligne], 3 | 1990, mis en ligne le 19 avril 2011, consulté le 06 mai 2019. URL : http://journals.openedition.org/kernos/967 


\section{MARIE DELCOURT, DIX ANS DÉJÀ...}

\section{Allocution d'hommage prononcée par André MOTTE, professeur à l'Université de Liège, au cours d'une soirée publique organisée, le 17 mars 1989, dans le cadre du Colloque.}

Au cours de ces journées, et ce soir plus particulièrement, une grande dame vit dans le souvenir de beaucoup d'entre nous. Il y a tout juste dix ans - c'était le 11 février 1979 - que Marie Delcourt nous a quittés. Comment ne pas saisir l'occasion de ce colloque pour lui rendre un nouvel hommage ? C'est, en effet, la première fois, je pense, que se tient à Liège une rencontre internationale consacrée à la religion grecque. Or Liège n'est pas seulement la ville où Marie Delcourt a fait ses études et a accompli toute sa carrière universitaire, c'est aussi une ville qu'elle a aimée et qu'elle a célébrée par son talent d'écrivain. Quant à la religion grecque, chacun sait qu'elle fut l'un des domaines de prédilection de ses recherches; chacun sait aussi combien elle l'a brillamment illustré et fait apprécier d'un très large public. Plus précisément encore, comment le thème Oracles et mantique choisi pour ce colloque pourrait-il ne pas évoquer le bel ouvrage qu'elle a consacré, en 1955, au sanctuaire oraculaire le plus illustre de la Grèce antique, celui de Delphes ? Et il n'est pas jusqu'au titre de la conférence que va faire François Jouan - L'oracle, thérapeutique de l'angoisse - qui ne rappelle l'enrichissement considérable que Marie Delcourt, l'une des premières, a apporté à nos études en prêtant intérêt à la dimension psychologique des faits religieux.

C'est dire déjà que notre Université s'honore de l'avoir comptée parmi ses maîtres et que ce n'est pas un hasard s'il se trouve encore à Liège, aujourd'hui, des hellénistes que ce domaine d'étude continue de passionner et qui se réjouissent que l'occasion se présente de pouvoir dire publiquement : «merci, Marie Delcourt».

Mais il n'est certes pas que des hellénistes à avoir recueilli son héritage. Son œuvre fut, en effet, d'une très riche diversité, et elle surprend par son ampleur : plus de trente volumes et une centaine d'articles. Il n'est guère de période de l'histoire qui n'ait suscité la curiosité de Marie Delcourt. Toute sa vie, elle a pratiqué le métier exigeant de philologue, éditant de nombreux textes, comme l'Utopie de Thomas More et la correspondance d'Érasme, cultivant aussi, avec un 
rare bonheur, l'art de la traduction; celle qu'elle a consacrée aux tragédies d'Euripide, pour ne prendre qu'un exemple, vient de connaître une troisième édition. Ses travaux de critique littéraire couvrent un large champ qui va des Tragiques grecs jusqu'à Claudel, en passant par Platon et Plutarque, Ménandre et les Comiques latins, Horace et Virgile, Molière, Goethe et bien d'autres encore. Historienne, elle affectionne particulièrement les monographies : Euripide, Eschyle, Périclès, Érasme, mais elle s'intéresse aussi à l'hagiographie, à l'histoire des sciences, de la pensée politique, de la philosophie. Elle consacre des articles à l'organisation de l'enseignement, à la méthodologie et à divers sujets d'actualité. On lui doit aussi des souvenirs de voyage, Images de Grèce, un ouvrage sur Liège et même un livre de cuisine. Et j'en passe.

Cette œuvre témoigne assurément d'une ouverture d'esprit hors du commun. Au premier abord, elle pourrait même paraître composite, mais elle trouve son unité profonde dans le dessein humaniste qui l'inspire : une attention sans cesse en éveil pour tout ce qui est humain, pour l'homme du passé, le plus souvent, mais en ce qu'il signifie encore à l'homme d'aujourd'hui, une longue méditation aussi sur l'existence humaine, sur les situations tragiques et, plus spécialement, sur les disgrâces initiales du sort, celles qui marquent souvent les destinées héroïques. Humaniste, son œuvre l'est encore par ses qualités esthétiques et littéraires : la sobre élégance de son style est celle d'un savant doublé d'un artiste.

Jalonnant toute la production scientifique de Marie Delcourt, les très nombreuses études consacrées à la religion grecque illustrent remarquablement ces préoccupations qui n'ont cesser de l'animer. Ce n'est pas un hasard si elle aimait parler d' «humanisme religieux" pour caractériser cette composante, essentielle à ses yeux, de la culture antique. À défaut de pouvoir passer ici en revue chacun des ouvrages marquants, je voudrais tenter d'esquisser globalement ce qui me parait caractériser leur démarche.

C'est avec une curiosité exigeante, mue aussi par une sympathie préalable que Marie Delcourt aborde l'étude des faits religieux. «Quelque chose de nouveau a commencé», m'écrivait-t-elle en 1973, «quand la raison a considéré comme des objets dignes d'elle les produits de l'imagination, ne les prenant plus pour des lubies gratuites, mais pour des faits significatifs, qui méritaient qu'elle leur appliquât ses rigoureuses méthodes». À l'époque où elle entreprenait ses premières investigations sur les cultes et les mythes, pareilles conceptions étaient loin de prévaloir dans toutes les écoles de philologie 
classique. Il est significatif, d'autre part, qu'elle ait choisi de consacrer deux ouvrages de synthèse à l'étude de la vie religieuse telle qu'elle s'organisait, avec toute sa luxuriance, dans les grands centres : Les grands sanctuaires de la Grèce (1947), et L'oracle de Delphes (1955). Car une des idées maîtresses qu'elle entend de la sorte illustrer, c'est que les récits sacrés, les croyances et les spéculations religieuses, d'une part, les cultes, les pratiques rituelles et les institutions, d'autre part, entretiennent, au niveau des représentations mentales, des rapports complexes qu'il faut tenter de sonder afin de les mieux comprendre. L'étude des faits religieux ne peut se contenter d'inventaires cloisonnés. Dans ce sens, un autre ouvrage de synthèse, Légendes et cultes des héros en Grèce, consacrait déjà, en 1942, tout un chapitre, méthodologiquement important, à montrer «la substance religieuse des légendes».

Douée, dès le départ, d'une sensibilité très fine pour la compréhension du phénomène religieux, Marie Delcourt s'est appliquée à en explorer les composantes diverses : genèse et évolution, aspects sociaux et psychologiques aussi, avec le souci cependant de ne verser dans aucune forme de réductionisme.

C'est plus spécialement dans le domaine des mythes et des légendes qu'elle a déployé son art subtil de l'analyse, reprenant à nouveaux frais des thèmes déjà connus, comme celui du parricide, de l'inceste, de l'exposition d'enfants (Edipe) ou du matricide (Oreste et Alcméon), ou bien faisant surgir de la confrontation de témoignages épars des sujets neufs, comme celui de la monstruosité (Stérilités mystérieuses), du dieu lieur et magicien (Héphaistos), de l'ambivalence sexuelle (Hermaphrodite), du couple divin du Roux et de la Rousse (Pyrrhos et Pyrrha). Mais l'originalité de ces recherches ne tient pas seulement au choix des thèmes ou à une documentation patiemment renouvelée; elle est dans la méthode d'exégèse que leur auteur s'est progressivement forgée, une méthode qu'il est d'ailleurs malaisé de cerner en quelques mots, car elle se veut souple et répugne au dogmatisme. «En de telles matières", disait-elle, «il est impossible de dépasser la simple probabilité». C'est une leçon de modestie que nous pouvons encore méditer.

Anticipant, par certains côtés, sur l'approche structurale, Marie Delcourt prend soin d'abord de recueillir toutes les versions des mythes qu'elle étudie, car "un mythe n'est jamais terminé». Elle en scrute les variantes les plus significatives pour mieux en dégager les noyaux constitutifs. L'examen des contextes sociaux, - la référence aux pratiques cultuelles notamment, - aide à décrypter ces ensembles. Linterrogation sur le sens des mythèmes fait volontiers place aussi à 
des considérations d'ordre psychologique. Dans les mythes et les légendes, l'âme humaine dévoile certaines tendances profondes et permanentes. Marie Delcourt se réclame des travaux de Bachelard et fait aussi un usage mesuré des concepts jungiens d'archétype et d'inconscient collectif. Mais elle est fort peu encline à appliquer les concepts rigides et les théories réductrices d'une psychanalyse orthodoxe. On le voit notamment à propos de son interprétation du mythe d'EEdipe. Elle considère, en définitive, que "les tendances subconscientes sont plutôt des collaboratrices de la mémoiren; elles ne créent pas à proprement parler le mythe.

Le fait de mettre ainsi l'accent sur les questions de méthode auxquelles les ouvrages de Marie Delcourt accordent une attention soutenue ne doit pas faire oublier leur grande richesse de contenu. On ne les lit, on ne les relit jamais sans profit, tant ils regorgent de faits, d'analyses pénétrantes, de rapprochements suggestifs qui stimulent la réflexion. Au besoin, lorsque les témoignages grecs ne fournissent pas la lumière escomptée, l'auteur sait puiser dans le patrimoine de la mythologie et du folklore universels les éléments d'une comparaison éclairante. Mais elle ne le fait jamais qu'avec prudence.

Bref, dans son effort persévérant pour mieux faire connaître et comprendre la religion des Grecs, comme en d'autres domaines aussi, Marie Delcourt fait figure de précurseur avisé. C'est pourquoi son œuvre présente aujourd'hui si peu de rides. Et tel est aussi le souvenir que conservent de ce maître vénéré les nombreux amis et anciens élèves qui ont bénéficié de sa grande générosité d'esprit et de cœur.

Car l'œuvre scientifique de celle qu'entre eux ses étudiants appelaient affectueusement «Marie» n'épuise pas sa riche personnalité. Aussi bien cette assemblée ne regroupe-t-elle pas seulement d'anciens collègues ou étudiants, des lecteurs admiratifs, des passionnés d'hellénisme et d'humanisme, mais aussi de vrais amis. Ils viennent, voici quelques semaines, de se réunir autour de son époux, l'écrivain Alexis Curvers, pour fonder une association des «Amis de Marie Delcourt" ${ }^{1}$. Puisse cette hétairie largement ouverte contribuer à perpétuer le souvenir de la grande dame en diffusant son œuvre et son message d'humanisme.

1 Adresse de l'Association : Quai Churchill, 19, bte 091, B - 4000 Liège. 
Bibliographie des œuvres de Marie Delcourt se rapportant à la religion et à la mythologie grecques

Outre une série d'articles consacrés à des héros, - Héraclès, Sarpédon, Néoptolème, etc., - relèvent directement du domaine les ouvrages suivants :

- Stérilités mystérieuses et naissances maléfiques dans l'antiquité classique, Paris-Liège, Les Belles Lettres-Bibliothèque de la Faculté de Philosophie et Lettres de l'Université de Liège, 1938; 2e éd., 1986;

- Légendes et cultes des héros en Grèce, Paris, P.U.F., 1942;

- Edipe ou la légende du conquérant, Paris-Liège, Droz-Bibliothèque de la Faculté de Philosophie et Lettres de l'Université de Liège, 1944; 2e éd., Paris-Liège, Les Belles Lettres-Bibliothèque de la Faculté de Philosophie et Lettres de l'Université de Liège, 1981 (Confluents psychanalytiques);

- Les grands sanctuaires de la Grèce, Paris, P.U.F., 1947; 2e éd., Brionne, Gérard Montfort Éditeur, 1982;

- L'oracle de Delphes, Paris, Payot, 1955; 2e éd., 1981;

- Héphaistos ou la légende du magicien, Paris-Liège, Les Belles LettresBibliothèque de la Faculté de Philosophie et Lettres de l'Université de Liège, 1957; 2e éd., 1982 (Confluents psychanalytiques);

- Hermaphrodite. Mythes et rites de la bisexualité dans l'antiquité classique, Paris, P.U.F., 1958; trad. angl. : Hermaphrodite. Myths and Rites of the Bisexual Figure in Classical Antiquity, London, Studio Books, 1961;

- Oreste et Alcméon. Étude sur la projection légendaire du matricide en Grèce, Paris-Liège, Les Belles Lettres-Bibliothèque de la Faculté de Philosophie et Lettres de l'Université de Liège, 1959;

- Images de Grèce, Bruxelles, 1943; 2e éd., Namur, Wesmael-Charlier, 1959 ;

- Pyrrhos et Pyrrha. Recherches sur les valeurs du feu dans les légendes helléniques, Paris-Liège, Les Belles Lettres-Bibliothèque de la Faculté de Philosophie et Lettres de l'Université de Liège, 1965; 
- Hermaphroditea. Recherches sur l'être double promoteur de la fertilité dans le monde classique, Bruxelles, Latomus, 1966.

\section{Hommages et études}

- Hommages à Marie Delcourt, Bruxelles, Latomus, 1970; le volume comprend (p. 9-19) une bibliographie, due à Marcelle Derwa, des publications de Marie Delcourt jusqu'en 1969;

- Un numéro de la revue Critique (293, oct. 1971) consacre à Marie Delcourt quatre articles parmi lesquels on notera la remarquable étude d'H. Rousseau sur Les mécanismes de la pensée mythopoétique des Grecs;

- Actes du Colloque Hommage à Marie Delcourt (Université de Liège, 5 nov. 1982), Liège, Faculté Ouverte, 1983, comprenant des contributions de J. Labarbe, R. Crahay (Marie Delcourt, historien des religions), F. Bierlaire, J. Hoyoux et A. Curvers. 